endometriosis compared to after surgery of a IV stage of endometriosis $(p<0,05)$. From all analysed deliveries $31,25 \%$ ended with cesarean section. More deliveries via cesarean section were found after IVF compared to natural conception $(p>0,05)$. A higher frequency of cesarean section was found in women who got pregnant after IV stage endometriosis surgery compared to those after III stage surgery $(p>0,05)$.

Conclusions Women after a surgery of III stage endometriosis have an increased probability of pregnancy complications compared to woman after surgery of IV stage endometriosis. Rate of cesarean section is still increased after a surgery of advanced endometriosis. Cesarean sections are performed more frequently on women after surgery of IV stage endometriosis than after surgery of III stage endometriosis.

\section{HOW ELECTRONIC PRESCRIBING BUNDLES IMPROVED PAIN RELIEF PRESCRIBING PRACTICES FOR PATIENTS UNDERGOING ELECTIVE CAESAREAN SECTIONS}

J Patch*, V Pattni, S Bell. Princess Alexandra NHS Trust, Harlow, UK

\subsection{6/rapm-2021-ESRA.120}

Background and Aims The elective caesarean section (CS) rate in England was $15 \%$ in 2019. It is vital to offer adequate pain relief to these patients to improve experience and reduce morbidity. NICE suggests intrathecal/epidural diamorphine for analgesia. If no contraindications, NSAIDs should be offered. RCoA advises that women receiving opioids should have pain scores recorded and anti-emetics and laxatives prescribed.

The prevalence of electronic prescribing in UK hospitals is increasing. These systems can be beneficial in improving patient safety, governance and productivity.

We audited compliance with aforementioned standards and instigated changes to electronic prescribing systems to improve practice.

Methods Data collected prospectively for 26 patients using anaesthetic records, online prescribing charts and pain assessment by questioning patients. Objectives were; 100\% women satisfied with analgesia day 1 post CS; 100\% women receive intrathecal/epidural opioids with regional anaesthetic; Unless contraindicated, 100\% women prescribed NSAIDs; 100\% women prescribed anti-emetics and laxatives PRN.

The cycle was repeated with 15 patients following alteration of prescribing bundles to include recommend medications. Results Initially, $100 \%$ of patients given intrathecal opioid. $69 \%$ received NSAIDs. $19 \%$ prescribed laxatives and $96 \%$ prescribed anti-emetics. $100 \%$ of women expressed satisfaction with pain relief. After modification, opioid use remained $100 \%$. NSAID use rose to $100 \%$. $80 \%$ prescribed laxatives

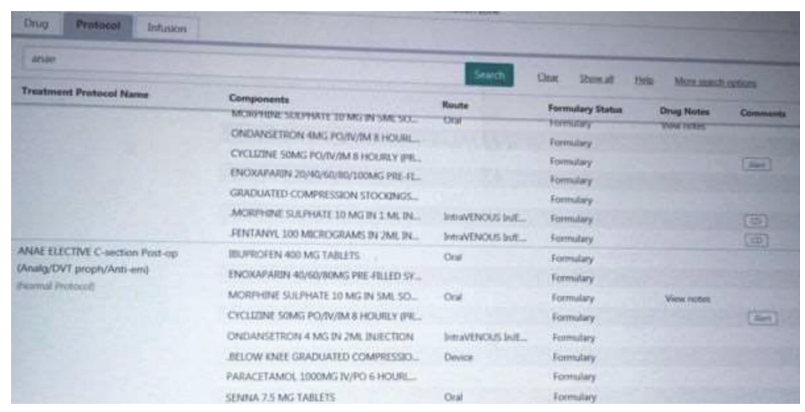

Abstract 120 Figure 1 and $100 \%$ prescribed anti-emetics. Satisfaction remained $100 \%$.

Conclusions Adequate post-operative analgesia is a cornerstone of good anaesthetic practice. Electronic prescribing bundles improved compliance with national standards of recommended analgesia for patients undergoing elective CS.

\section{POSTPARTUM POSTDURAL PUNCTURE HEADACHE: WHEN THINGS CAN GO WRONG..}

K Theodoraki*, I Siafaka. Aretaieion University Hospital, National and Kapodistrian University of Athens, Athens, Greece

\subsection{6/rapm-2021-ESRA.121}

Background and Aims We report a case of cerebral venous sinus thrombosis (CVST) after no obvious dural puncture in a postcesarean section patient

Methods A 32-year old primigravida underwent cesarean section under a combined spinal-epidural. She had a medical history of panic attacks and depression. On the second postpartum day, the parturient developed orthostatic headache which was considered as postdural puncture headache and treated accordingly. Three days postdischarge, the headache returned, having lost its orthostatic component. The parturient however refused to return to hospital for reassessment. Over the next few days, the parturient started behaving strangely and refusing interaction with the newborn. A psychiatrist consulted by the family diagnosed anxiety disorder based on the parturient's previous medical history and suggested anti-anxiety medication.

Results On the seventh postpartum day, the parturient was urgently readmitted to hospital, unable to speak and looking catatonic. Major postpartum depression was diagnosed by the attending psychiatrist and antidepressive medication was initiated. However, on the first readmission night, the parturient developed tonic-clonic seizures and an urgent brain CT-scan revealed multiple left-sided infarcts with haemorrhagic foci. CVST was diagnosed, low-molecular weight heparin was started and the parturient's situation improved dramatically

Conclusions CVST is attributed to cerebral venous dilation and intracranial hypotension in the setting of postpartum hypercoagulability and can have a deleterious outcome if unsuspected and untreated. This case of misdiagnosis emphasizes the need for urgent neurological consultation in the puerperium in case of new or recurrent neurological symptoms. CVST should be considered in the differential diagnosis, especially in case of atypical clinical presentation.

\section{EPIDURAL BLOOD PATCH IN THE $3^{\text {RD }}$ TRIMESTER OF PREGNANCY. WHAT ABOUT LABOUR ANALGESIA?}

P Rebelo, AS Nogueira, C Pinto*. Hospital Pedro Hispano, Matosinhos, Portugal

\subsection{6/rapm-2021-ESRA.122}

Background and Aims Postdural puncture headache (PDPH) is a well-known and common complication of spinal anesthesia which depends upon the characteristics of spinal needle.

Methods We report a blood patch in the 3rd trimester of pregnancy. 